\title{
ANALYSIS OF ECONOMIC LOAD DISPATCH USING FUZZIFIED PSO
}

\author{
Bhagyashree Hosamani ${ }^{1}$, C R Sharada Prasad ${ }^{2}$ \\ ${ }^{1}$ M.Tech Student, EEE, UBDT College of Engineering, Davanagere, Karnataka, India \\ ${ }^{2}$ Associate Professor, EEE, UBDT College of Engineering, Davanagere, Karnataka, India
}

\begin{abstract}
With large interconnection of the electric networks, the energy crisis in the world and continuous rise in prices, it is very essential to reduce the running charges of the electric energy i.e., reduce the fuel consumption for meeting a particular load demand. The main aim in the economic dispatch is to minimize the total cost of generating real power at various stations while satisfying the loads and the losses in transmission links. A novel method is introduced in the planning and operation of power systems to provide reliable and quality power to consumers at economical cost. The primary objective of this paper is to develop efficient and fast Fuzzified Particle Swarm Optimization (FPSO) algorithm that can be applied to obtain the optimal solutions of multi-constrained dynamic ED, OPF. In this paper, I considered Fuzzified particle swam optimization to solve multi-constrained dynamic economic dispatch problem. The FPSO Method is inferred to have superior features than conventional methods and gives faster and stable convergence characteristics. PSO is popular due to its significant property of dealing with optimization problem and ease of computation. But the critical drawback of PSO is more number of iterations required and hence the computation time is required to obtain the optimal solution is more and also the premature convergence Hence to overcome the premature convergence and to speed up the process, a classical PSO technique is incorporated with fuzzy logic to get a faster convergence This improved technique is termed as Fuzzified Particle Swarm Optimization (FPSO). The proposed method can provide an accurate solution with fast convergence and has the potential to be applied to other power system optimization problems.
\end{abstract}

Keywords: Economic Dispatch(ED), Optimal Power Flow (OPF), Particle Swarm Optimization (PSO).

\section{INTRODUCTION}

In electrical power system operation we can minimize the total generation cost by solving the economic dispatch problem by satisfying some constraints. Economic load dispatch is taken as optimization problem, to minimize the generation cost. There are many algorithms to find the rate of optimum product for every power generation division. Usual algorithms that are used to solve the economic load dispatch problems are,

- Lambda iteration method.

- Gradient method.

- Newton method.

Demand on energy is increasing day by day, whereas energy resources are decreasing on the other side. Thus optimization is necessary for power system operation and planning. Economic Dispatch provides the best optimization scheme for selecting the best generation schedule for supplying a preset load, with minimum cost and satisfying the constraints [1]. The computation of optimum generation schedules for the predicted loads over a period of time, with due considerations of generator ramping rate limits, non-smooth fuel cost function due to valve-point effect, spinning reserve contribution constraints and prohibitive operating zones leads to an advanced economic dispatch with multiple objectives and several adequate constraints, effectively termed as multiconstrained dynamic economic dispatch [2]. Thermal power stations are a major cause of atmospheric pollution, due to the high concentration of pollutants emitted. Currently, with the increasing awareness of environmental pollution caused by burning fossil fuels, controlling the amount of pollutant emission is also a major criterion in the economic dispatch problem. The purpose of Emission Constrained Economic Dispatch (ECED) is to obtain the optimal generation schedule by minimizing the fuel cost and the emission level simultaneously while satisfying load demand and other operational constraints [3].

From the exhaustive literature review on OPF it is inferred that numerous deterministic and stochastic optimization techniques have been applied for solving the OPF problems. Moreover the conventional deterministic mathematical optimization techniques are complex and leads to high computational burden for large scale systems, and for stochastic algorithms the large number of iterations leads to a very large computation time. Partial Swarm Optimization (PSO) technique is superior over all other stochastic techniques cited in the literature review. But certain critical Limitations are observed in the general PSO technique. The literature review reveals that there exists a need for developing simple, effective and accurate algorithm for solving ED and OPF problems without and with security constraints. Hence, in this research, an amendment has been made to PSO algorithm to overcome drawbacks [2][3][5]. 
In this paper a new method is introduced to solve economic load dispatch problem so that the state of the present power system can be optimized. the various objectives considered in the optimization problem are minimization of total generation cost, minimization of total network active power loss and maximization of security level.

\section{ECONOMIC LOAD DISPATCH}

In electric power industry the importance is given to the operation of electric power system optimally and efficiently. Now a day, the utilities are trying to minimize the cost of running power systems and also trying to satisfy the demand of their customers and by doing so the profit can be achieved. For committed units it is necessary to operate optimally to supply the expected load demand of customers even with limited number of generating units, increasing fuel cost and also increasing demand for power in different time intervals. The main objective in ELD is to schedule the outputs of committed generating units by meeting the power demand with reduced operating cost to the utilities and also satisfying equality and inequality constraints. Previously many researches have been carried out for load dispatch problem. There are different problems involved in ELD, the two main problems considered are, Unit Commitment or pre-dispatch problem and On-line economic dispatch. In unit commitment problem the optimal generating unit is selected among the available generating units to meet the expected load demand. In the on-line economic dispatch the load is distributed equally among the generating units in order to minimize the total cost of supplying power [10].

\subsection{Conventional Methods for solving ELD Problem}

1. The Lambda-Iteration Method

2. The Gradient Search Method

3. Newton's Method

4. ED with Piecewise linear Cost Functions

5. Base Point \& Participation factor.

6. Linear Programming.

7. Dynamic Programming.

Fuzzy Logic and Particle Swarm Optimization methods are introduced recently for solving load dispatch problem.

\section{ECONOMIC LOAD DISPATCH USING FUZZY LOGIC}

The fuzzy logic method is treated as robust method because it deals with fuzzy or crisp values and does not require precise inputs and it can be programmed even if a feedback sensor quits or is destroyed. Even though there is a wide variation in the inputs the output control is a smooth control function. The fuzzy logic controller processes user-defined rules hence it can be modified easily to improve or modify system performance. By the generation of appropriate governing rules the system can be incorporated with new sensors. Fuzzy logic is not limited one or two control outputs, and it is not necessary to compute rate-of-change of parameters in order for implementation. The sensor data is sufficient because that provides some indication of a system's actions and reactions. Hence the sensors will be inexpensive and less complex [3].

\subsection{Advantages \& Disadvantages:}

\subsubsection{Advantages:}

1. ANFIS (Adaptive Neuro-Fuzzy Inference System) method is faster in operation and gets better results in most cases compare to delta gradient and analytical method.

2. ANFIS Method uses the MATLAB toolbox and it is applied to solve the problem that involves large number of power plants.

3. Fuzzy logic solutions are easy to verify and optimize.

\subsubsection{Disadvantage:}

The variation in the limits of the real power generated will be within minimum and maximum. Hence in the real time optimization ELD problem will become more non-linear.

\section{ECONOMIC LOAD DISPATCH USING PSO}

Particle swarm optimization (PSO) is a technique of solving optimization problem which considers the population based stochastic optimization technique. This method is developed by Dr.Ebehart and Dr. Kennedy in 1995, the main inspiration is by the social behavior of bird flocking or fish schooling. PSO is a robust, stochastic computational technique based on movement and intelligence of swarm. PSO has many similarities with evolutionary computation techniques such as Genetic Algorithms (GA). The initialization of the system is done with a population of random solutions and by updating generations it will search for optimum solution. In GA approach there are evolution operators such as crossover and mutation. But PSO has no such operators. In PSO, the particles fly through the problem space by following the current optimum particles. PSO is easy in implementation and having less parameter to make adjustment as compared to GA. This is one of the advantages of PSO. The many areas of application of PSO are: function optimization, artificial neural network training, fuzzy system control, and other areas where GA can be applied [6].

\subsection{Problem Formulation [9]:}

$$
\begin{aligned}
& V_{i}^{(u+1)}=\mathrm{w}^{*} V_{i}^{(u)}+\mathrm{C} 1 * \text { rand }() *\left(\text { pbest }_{i}-P_{i}^{(u)}\right)+\mathrm{C} 2 * \text { rand } \\
&()^{*}\left(\text { gbest }_{i} P_{i}^{(u)}\right) \\
& P_{i}^{(u+1)}=P_{i}^{(u)}+V_{i}^{(u+1)}
\end{aligned}
$$


Where $\mathrm{C} 1, \mathrm{C} 2$ are constants

The inertia weight ' $W$ ' is set according to the following equation,

$$
W=W_{\max }\left[\frac{W_{\max }-W_{\min }}{{ }^{2} E R_{\max }}\right] \times I T E R
$$

\subsection{Steps of Implementation:}

1. Initialize the total cost function this is also called as Fitness Function. The total cost function is derived from the individual cost function of the various generating stations.

2. Initialization of the Parameters of the PSO they are Population size, $\mathrm{C} 1, \quad \mathrm{C} 2, W_{\max }, W_{\min }$, error gradient etc.

3. Giving the inputs such as the Fuel cost Functions, MW limits of the generating stations along with the B-coefficient matrix and the total power demand.

4. In the first step the random allocation of large number of vectors (equal to the population size) of active power are done by satisfying the MW limits.

5. The value of fitness function is calculated for each vector of active power. The values obtained in the iteration are compared to obtain Pbest. At each iteration all values of the whole population are compared to obtain the Gbest. These values are updated at each step.

6. The error gradient is checked at each step and the value of Gbest is plotted till it comes within the prespecified range.

7. The final value of Gbest is the minimum cost and the active power vector represents the economic load dispatch solution.

\subsection{Disadvantages:}

1. Since PSO based algorithms are random search techniques, the convergence will be very slow and inconsistent for a large scale interconnected power system as there are enormous number of decision variables.

2. The inertia weight (in velocity adaptation) in PSO is fixed throughout the whole search process. However, for practical applications a small fixed inertia weight may result in a premature convergence. Hence there is a need for an adaptive inertia weight.

\subsection{Need for Fuzzified PSO}

1. PSO algorithm has very large computation time due to the large number of iterations required to obtain a global optimum.

2. In classical PSO technique the value of inertia weight computation is completely based on iteration count which leads to slow and premature convergence. Hence there is a need for an adaptive inertia weight.

\section{FUZZIFIED PARTICLE SWARM OPTIMIZATION BASED ECONOMIC LOAD DISPATCH}

This presents an overview of some superior stochastic optimization techniques followed by the need and formulation of Fuzzified Particle Swarm Optimization (FPSO) algorithm for solving Multi-constrained Dynamic Economic Dispatch, Emission Constrained Economic Dispatch (ECED) and Multiarea ED problems. Subsequently the FPSO algorithm is applied on standard test systems for solving the above mentioned ED problems and the optimal results obtained are compared and analyzed with other stochastic optimization techniques[7][8].

\subsection{Problem Formulation:}

Step 1 (Initialization): An initial swarm of particles $I i$ of size $n$ is generated randomly within the feasible range and the distributions of initial trial parents are uniform. The elements of each initial particle are the controllable real power outputs of committed $n g$ generating units.

$$
\begin{aligned}
& I_{i}=\left[P_{G 1}^{t i}, P_{G 2}^{t i}, \ldots \ldots=P_{G j}^{t i}, \ldots \ldots \ldots=P_{G n g}^{t i}\right] ; i=1,2 \ldots n ; \\
& t=1,2 \ldots N T
\end{aligned}
$$

Step 2 (Velocity updating): Using the Pbest and Gbest compute the $i$ th particle velocity as,

$$
\begin{aligned}
& v_{k}^{i \text { iter+1 }}=\chi\left\{w \times v_{k}^{i \text { iter }}+c_{1} \times \text { rand }\left(\text { Pbest } t_{j}^{i}-x_{k}^{i \text { iter }}\right)+\right. \\
& \left.c_{2} \times \text { rand }\left(\text { Gbest }{ }_{j}-x_{k}^{i \text { iter }}\right)\right\}
\end{aligned}
$$

Step 3 (Position updating): Based on the updated velocities, each particle changes its position as,

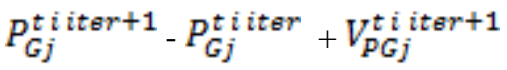

If a particle violates its position limits in any dimension, set its position at the violating limit.

Step 4 (Updating Pbest and Gbest): With the updated position evaluate fitness of all particles in the swarm and obtain the Pbest of each particle and thereby compute the Gbest.

Step 5 (Stopping criteria): If the preselected maximum iteration is reached then the Gbest is the global optimal solution, otherwise increment the iteration count and repeat steps 2 to 4 [2]. 
The above algorithmic steps are depicted in the Flow Chart below.

\subsection{Flowchart}

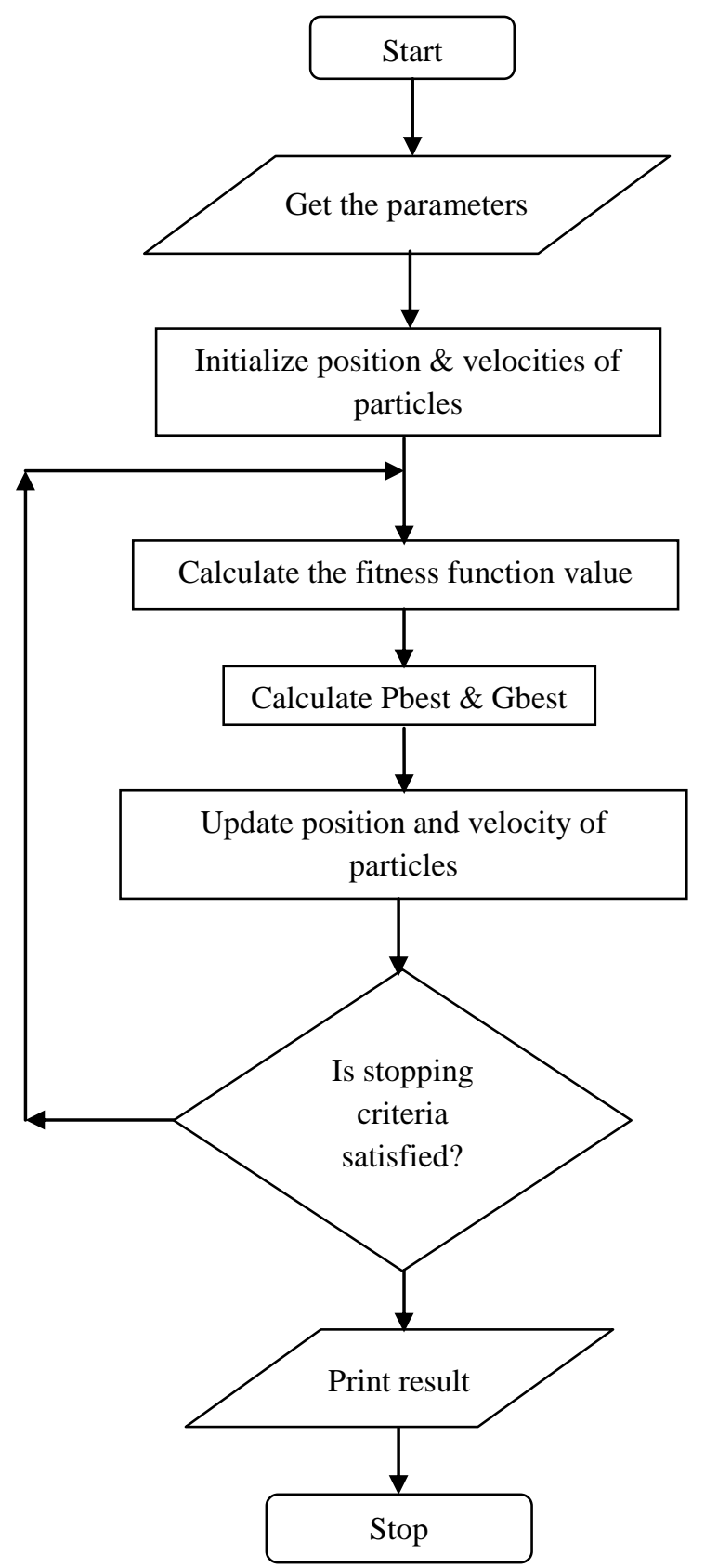

Fig - 1: Flow chart for Fuzzified PSO based ELD.

\section{RESULTS}

A program has been developed in Matlab and results are obtained for Fuzzified PSO. Graphs show less iterations and less computational time. Graphs are shown below (Fig 2 and Fig 3).

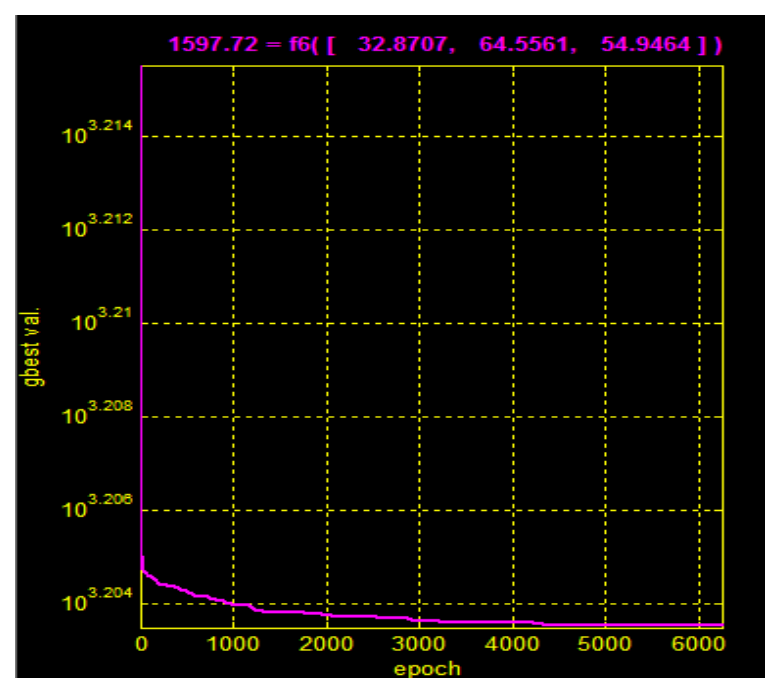

Fig - 2: Graph of Gbest value versus epoch

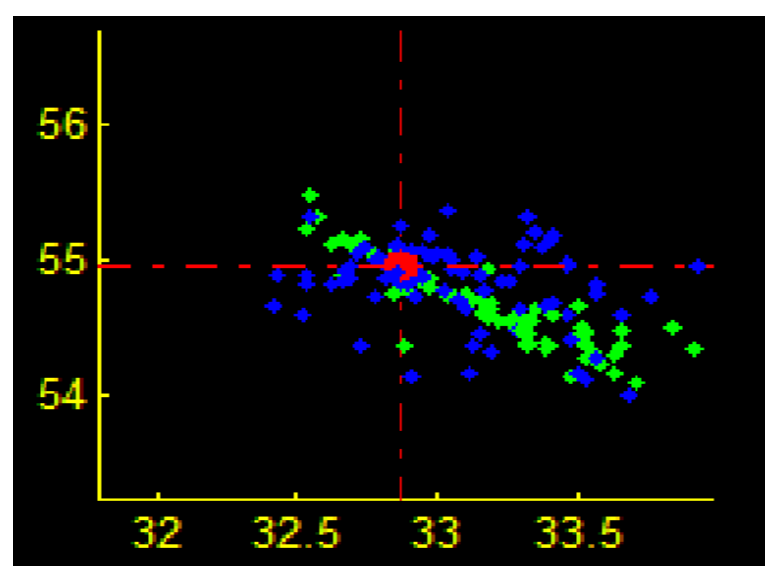

Fig- 3: Convergence Characteristics

Green color represents the personal best (Pbest), and red color represents the global best (Gbest) among the particles in Fig 3 .

\section{CONCLUSIONS}

The primary objective of this paper is to develop efficient and fast Fuzzified Particle Swarm Optimization (FPSO) algorithm that can be applied to obtain the optimal solutions of multiconstrained dynamic ED, ECED, multi-area OPF, multi-area Security Constrained OPF (SCOPF) and OPF with multiple FACTS controllers.

An enhanced ED problem is developed considering all the practical constraints leading to an advanced economic dispatch with multiple objectives and constraints. A new improved stochastic technique namely Fuzzified Particle Swarm Optimization (FPSO) is applied to solve multi-constrained dynamic ED problem. 
The proposed FPSO method is used for solving emission constrained economic dispatch problem. It is a bi-objective problem.

The FPSO method is inferred to have superior features than the classical Particle Swarm Optimization (PSO) methods such as faster and stable convergence characteristics.

The Fuzzified Particle Swarm Optimization algorithm developed will be extremely useful for electric power utilities, for enhancing the economy and security of operation in their systems.

\section{REFERENCES}

[1]. Chowdhury, B.H. and Rahman, S. "A review of recent advances in economic dispatch," IEEE Transactions on Power Systems, Vol. 5, No. 4, pp. 1248-1259,1990.

[2]. Mohd. Asif Iqbal. "Analysis and Comparison of Lambda Iteration, Genetic Algorithm and Particle Swarm Optimization to Solve Economic Load Dispatch Problem" IASIR-IJSWS 2012

[3]. A. Laoufi, A Hazzab and M. Rahli, "Power Dispatch Using Fuzzy-Genetic Algorithm.”IJAER 2009

[4]. T. Ratniyomchai, A. Oonsivilai, P. Pao-La-Or, And T. Kulworawanichpong, "Particle Swarm Optimization for Solving Combined Economic and Emission Dispatch Problems."RAEE Thailand

[5]. M.A. Abido, "Multiobjective particle swarm optimization for environmental/economic dispatch problem." Electric Power Systems Research 79 (2009) 1105-1113.

[6]. Rasoul Rahmani, Mohd Fauzi Othman "Solving Economic Dispatch Problem Using Particle Swarm Optimization By An Evolutionary Technique For Initializing Particles". JATIT, 2012

[7]. T. Ratniyomchai, A. oonsivilai, "Particle Swarm Optimization for Solving Combined Economic and Emission Dispatch Problems." Recent Advances In Energy \& Environment. 2010.

[8]. Taher Niknam, Hasan Doagou Mojarrad and Majid Nayeripour. "A New Hybrid Fuzzy Adaptive Particle Swarm Optimization For Non-Convex Economic Dispatch." IJICICI, Volume 7, January 2011

[9]. N.M Jothi Swaroopan., P. Somasundaram, "Fuzzified PSO Algorithm For DC-OPF of Interconnected Power System" Journal of Theoretical and Applied Information Technology. 2010.

[10]. Mahesh Prasad Mishra, "Solution to Economic Load Dispatch Using PSO" National Institute of Technology, Rourkela 2012. 